\title{
Multi-dimensional database technology based on artificial intelligence
}

\author{
Yurong Liu*, Yang Bai, Xiongjun Li, and Hao Chen \\ Beijing Jinghang Research Institute of Computing and Communication, Beijing, China
}

\begin{abstract}
Artificial intelligence and database technology are both important fields of computer science. The research results show that more and more industries have a growing demand for artificial intelligence and database. The combination of these two technologies will certainly bring a broader prospect to computer application. This paper mainly discusses the necessity, importance and development of multi-dimensional database technology based on artificial intelligence, introduces the combination strategy of artificial intelligence and multidimensional database and the current research field, and gives the research direction.
\end{abstract}

\section{Basic concepts}

\subsection{Artificial intelligence}

Artificial intelligence, abbreviated as AI. It is a new technology science to research and develop the theory, method, technology and application system for simulating, extending and extending human intelligence. Artificial intelligence is a branch of computer science. It attempts to understand the essence of intelligence and produce a new intelligent machine that can respond in a similar way to human intelligence. The research in this field includes robots, language recognition, image recognition, natural language processing and expert systems.

\subsection{Multidimensional database}

Multidimensional database is to store data in an n-dimensional array, rather than in the form of records as a relational database. Therefore, it has a large number of sparse matrices, and people can observe the data through multidimensional views. Multidimensional database adds a time dimension. Compared with relational database, it has the advantages of improving data processing speed, speeding up response time and improving query efficiency.

\section{Connotation of application of artificial intelligence in multidimensional database technology}

\footnotetext{
*Corresponding author:408687144@qq.com
} 
Multi dimensional database technology based on artificial intelligence is a new technology. This technology can realize the distributed storage of multi-modal data (unstructured data, semi-structured data and structured data), at the same time, it can also store the data in the form of hypercube, and carry out real-time multidimensional analysis and query on the data. Traditional multidimensional database directly extracts dimension information from two-dimensional table, without considering the correlation between dimension information. Therefore, combined with artificial intelligence technology, it can realize the association analysis of multi-modal data and generate dimension information automatically.

Specifically, based on the requirements of efficient analysis, storage and processing of multidimensional data in the field of business intelligence (BI), the application research of multidimensional database technology based on artificial intelligence is carried out to realize the unified collection of multi domain heterogeneous data, efficient, real-time and automatic tagging, clustering, intelligent extraction of data information and semantic association, hypercube storage and online analysis OLAP, online analytical processing, etc. A multi-dimensional database prototype system based on artificial intelligence is designed to meet the needs of intelligent analysis and processing of massive data. The query behavior patterns and data characteristics of users are systematically learned. Cube data model is constructed by built-in machine learning algorithm. Model optimization is carried out continuously and query results are accurately generated for specific users. Through the function modules of artificial intelligence such as distributed algorithm engine, hybrid on-line analytical processing and distributed storage engine, multi-source heterogeneous data resources are integrated to realize data association, intelligent learning, reasoning and prediction, providing more complete and reliable predictive decision-making services for management decision-making and business operation end.

\section{Current situation and development trend}

\subsection{Foreign status and development trend}

\subsubsection{Multi-dimensional database based on relational model}

Multidimensional database based on relational database is realized by multidimensional analysis of relational database. The core is relational database, that is to express and store multidimensional data through relational structure. The multidimensional structure of this kind of multidimensional database is usually divided into two types: one is fact table, which is usually used to store data and keywords; the other is dimension table, which is usually used to store dimension information such as members, categories and levels of each dimension. Fact table and dimension table are linked together by primary key and foreign key, thus forming "star pattern". The hierarchy of some dimensions is complex. In order to avoid occupying too much storage space due to data redundancy, multiple tables are usually used to describe these dimensions, resulting in an extended "snowflake pattern" of "star pattern". Both star structure and snowflake structure are based on relational database to simulate multidimensional model structure, but simulation itself can not provide real multidimensional data analysis ability, only form a statistical report. Oracle express server, Microsoft SQL Server Analysis Services and DB2 OLAP server are typical multidimensional database products based on relational model. 


\subsubsection{Multi-dimensional database based on multidimensional array storage}

The multidimensional database based on multidimensional array storage stores data in the database in the structure of "Cube". Because of this special storage form, the data in multidimensional database can be rotated, sliced and sliced directly. This brings great advantages to the database operation and makes the query speed improve very fast. The core technology of this database is aggregation operation, mdx analysis, vector calculation, etc. This type of multidimensional database products include Oracle Essbase, IBM cogons, kylin, etc.

\subsection{Domestic status and development trend}

Compared with the diversity and maturity of the research of multi-dimensional database abroad, the domestic research on multi-dimensional database technology started late, and the research and application of multidimensional database is in a backward level, and the foundation of the research on the underlying algorithm, model and cube is relatively weak. At present, the mainstream database manufacturers in China are still in the research and application stage of traditional OLTP relational database and analytical MPP relational database, mainly used for data statistics and simple analysis, including intcbueolap, starring technology transwarparrgodb, etc.

In conclusion, the multi-dimensional database products at home and abroad need to pre quantify the dimension information of multidimensional data, then carry out vector calculation, high-dimensional cube distributed storage and other operations.

\section{Research objectives and main research contents}

\subsection{Research objectives}

In the era of rapid development of science and technology, aiming at the performance bottleneck of traditional storage technology in multidimensional analysis and large-scale computing, the paper studies the multi-dimensional database technology based on artificial intelligence, the distributed algorithm engine technology enhanced by artificial intelligence, the multi-dimensional data mixed online analysis and processing technology and the distributed storage engine technology, and finally constructs the algorithm engine The storage engine and multi-dimensional database can automatically mark and improve the speed of accessing and processing massive data. It can help users to improve the access of large-scale data sets to the depth insight level of extreme speed, help enterprises to digital transformation and upgrade the enabling industry, which has great economic benefit potential.

\subsection{Main research contents}

This part mainly focuses on the research objectives, analyzes the technical risks for each research content, and realizes the problems from the question, the analysis of the problems and the solution through the steps of "multi-dimensional database technology based on artificial intelligence", "realization of multi-dimensional database prototype system based on artificial intelligence" and "Application Research of multi-dimensional database technology based on artificial intelligence" The whole process of problem and practice application. 


\subsubsection{Research on multi-dimensional database technology based on artificial intelligence}

The research content will start from different types of multidimensional data, and study the weak supervision intelligent annotation technology based on multi knowledge inspired, and the construction technology of unified business level semantic layer, aiming at the characteristics of flow data, business data, user behavior data and log data oriented to specific business, to realize the distributed algorithm engine of artificial intelligence enhancement. Realize automatic annotation of various input data, realize unified business level semantic association under specific business scenarios, define dimension type and data content of multidimensional data, and provide dimension information for subsequent research; and calculate data cube, optimize data cube cube, store and optimize data cube based on multidimensional dimension information of data Research on the technology of the transformation and inquiry planning. According to the existing dimension information, the necessary dimension, hierarchical dimension and joint dimension are processed, and the data model is defined, the problem of updating data model, dimension disaster and super high-level dimension is studied and solved. The slicing and block cutting, drilling and rotation technology of cube cube are realized, which is the theoretical support of multidimensional data analysis. At the same time, the research on index design, planning calculation and query planning process technology of query planning is studied to realize $\mathrm{mdx}$ and SQL. Distributed storage is carried out according to the generated data hypercube, and the data mapping, distributed storage, storage compression optimization and multi-dimensional data storage optimization technology are studied.

\subsection{2 realization of multi-dimensional database prototype system based on Artificial Intelligence}

The research on the implementation technology of artificial intelligence enhanced distributed algorithm engine, the realization of automatic intelligent annotation of multi-source and multi-type data for specific business scenarios, the feature learning of artificial intelligence enhancement for user business data to enhance the accuracy of business data analysis, and the dimension information definition of multidimensional data are completed through unified business oriented semantic association; research and development The technology of multi-dimensional data mixed online analysis and processing is studied to realize multi-dimensional model multi-view and multi-level data display, which can make the data more intuitive display. Through the aggregation, segmentation and selection of data, the flexibility of data query and analysis can be improved, so as to summarize and subdivide the data from different layers and different angles, further meet the requirements of different business scenarios Task requirements: research the implementation of distributed storage engine technology, multi-dimensional data storage mechanism, index skills, locking level and other technologies to realize the distributed storage of multidimensional data, analyze the impact of cube block dimensions on the generation time, data storage space and data query speed of multidimensional database, and realize storage optimization and improve storage performance.

\subsubsection{Application research of multi-dimensional database technology based on artificial intelligence}

Combined with the previous research results, when the multi-dimensional database enhanced by artificial intelligence is realized, the application scope and applicable conditions of the system are defined, and the intelligent manufacturing and equipment 
quality data are selected for specific application to verify the applicability, ease of use and reliability of the system. According to the use of the system development, verify the correctness of the implementation scheme.

\section{Conclusion}

In a word, the multi-dimensional database technology based on artificial intelligence is not only a research topic in the computer field, but also a new research direction of the application of artificial intelligence. Artificial intelligence is gradually combined with general system technology, and multidimensional database technology is also close to the direction of special application fields, The development of the combination of artificial intelligence and multidimensional database technology is practical and application-oriented. New application fields and application requirements will promote the further development of technology. Large scale, complexity, integration and intelligence will be the development direction of future technology.

\section{References}

1. Han Jianchao, research on the combination of artificial intelligence and database technology. Electronic technology and information science, 1992

2. Wang Qianqian, OLAP modeling and application of telecommunication business intelligence system, master thesis of Capital University of economics and trade, 2009

3. Tao Minghui, Luo Xinxing, current situation and development trend of database technology, computer development and application, 1998

4. Gong Strider, design and implementation of enterprise annuity data warehouse, master's thesis, Hunan University, 2013

5. $\mathrm{Gu}$ Yonggen, review of the combination of artificial intelligence and database technology, Journal of Huzhou Normal University, 1996

6. Zhaoxiaqi, lailing, guobaopeng, the use of multidimensional technology in the knowledge warehouse oriented to the subject, 2006

7. Hu Changzhong, application and research of data warehouse technology in CRM, master's thesis, Sichuan University, 2004

8. Yangxuefeng, Likang, Zhang Ketong, implementation and performance tuning of online analysis and processing, computer system application, 2003 